(1)

1Oxford Centre for Respiratory Medicine and Oxford Respiratory Trials Unit, Oxford University Hospitals NHS Foundation Trust, Oxford, UK. 2 Respiratory Medicine Laboratory, Nuffield Dept of Medicine, University Of Oxford, Old Road Campus, Oxford, UK. Instituto and Hospital CUF Porto, Porto, Portugal. ${ }^{4}$ Centre for Research in Health Technologies and Information Systems, Faculty of Medicine, Porto, Portugal.

\title{
Targeted therapies for lung cancer: how did the game begin?
}

\section{Landmark papers in respiratory medicine}

Lung cancer is the leading cause of cancer death worldwide, causing 1.4 million deaths annually [1]. Patients with advanced stage nonsmall cell lung cancer (NSCLC) are usually managed with combinations of platinum-based chemotherapy and they have poor prognosis with a median survival of 8 months [2]. Chromosomal rearrangements and gene mutations that contribute to the constant activation of kinases play a crucial role in the process of carcinogenesis. During the past decade, knowledge gained from next-generation sequencing has radically affected lung cancer management and led to targeted therapies. To this end, the discovery of missing lung cancer driving mutations together with comprehensive understanding of the molecular basis of the disease have driven the introduction of novel therapeutic agents that specifically target cancer cells. In this article, we will present the landmark studies of epidermal growth factor receptor (EGFR), Kirsten rat sarcoma viral oncogene homolog (KRAS) and echinoderm microtubule-associated protein-like 4-anaplastic lymphoma kinase (EML4-ALK) pathways, which are the targets of the most common inhibitors that are used on everyday clinical practice.

\section{EGFR}

EGFR is a cell-surface receptor, which is a member of the ErbB receptor family, and was discovered in 1978. It is known that activation of the EGFR path- way stimulates cellular proliferation. In NSCLC, EGFR and its' ligands epidermal growth factor and transforming growth factor- $\alpha$ are regularly overexpressed [3, 4]. These findings led to formation of the hypothesis that EGFR could be used as a drug target, aiming to cease lung cancer growth. In 2003, the US Food and Drug Administration approved the administration of an EGFR inhibitor, gefitinib, that halts oncogenic signalling by targeting the tyrosine kinase domain of the receptor. Initial clinical trials revealed a notable inconsistency in the response of NSCLC patients to treatment [5, 6]. Although for the majority of patients, gefitinib was ineffective to control the disease, a specific treatment group (Asian ethnicity and never-smokers) showed a significant response. A meta-analysis of responders and nonresponders revealed that the response to gefitinib was associated with the presence of heterozygous somatic mutations of the EGFR tyrosine kinase domain. Based on this, genomic and transcriptomic sequencing studies verified that the presence of EGFR mutations grants vulnerability to gefitinib treatment. The heterozygosity of the EGFR mutations reveals that they have a dominant oncogenic effect $[5,6]$. It is well established that these lung cancer driving EGFR mutations enhance growth factor signalling and thus activate the oncogenic RAS pathway downstream of EGFR.

Erlotinib is another first generation EGFR tyrosine kinase inhibitor used in the armamentarium of NSCLC management. The distinct success of gefitinib and erlotinib has been accompanied
Cite as: Kanellakis NI Jacinto T, Psallidas I. Targeted therapies for lung cancer: how did the game begin? Breathe 2016; 12: 177-179.

@ERSpublications

Landmark studies on genetic alterations underlying NSCLC have led to tailored therapies http://ow.ly/4nqOPs 
by the observation that acquired resistance is developed after a median of 1 year of treatment [7]. Ongoing research is aiming to develop novel medications (second generation inhibitors) to overcome resistance by the broader inhibition of kinases. Several second generation inhibitors have been tested (e.g. afatinib and dacomitinib) with promising preclinical results.

\section{KRAS}

KRAS is a small GTPase protein that functions as a molecular switch, and thus communicates with downstream EGFR signalling. KRAS interacts with major cellular pathways involving mitogenactivated protein kinase, STAT and phosphoinositide 3-kinase. KRAS mutations lead to a constantly active protein product (GTPase) even in the absence of growth factor signalling. PDE $\delta$ is a protein that binds to KRAS and contributes to trafficking of the protein in the cytoplasm. Due the fact that it is one of the most frequent mutated genes in lung cancer, KRAS is appraised as one of the most important promising drug targets in lung cancer therapy. Hence, rigorous efforts have been made during the past 15 years to discover KRAS inhibitors.

Research has focused on blocking enzymes involved in post-translational RAS modifications, but with no clinically successful agents [8]. Eventually, in 2013, ZimMERMANN et al. [9] identified a small molecule, deltarasin, that inhibits PDE $\delta$ and blocks its binding to KRAS. In vitro experiments with pancreatic cancer cell lines validated the potential of deltarasin to reduce cellular proliferation and stimulate cellular death in a KRASdependent manner. In addition, in vivo experiments with nude mice bearing human pancreatic tumour cell xenografts suggested that the agent impairs tumour growth with a dose-dependent effect [9]. Specifically, inhibition of the oncogenic mutant KRAS signalling is feasible, leading to the reduction of tumour growth. Overall, as it is well established that patients bearing KRAS mutations do not respond to EGFR treatment, tumour genotyping is an emerging necessity for moving towards the optimal individualised treatment of NSCLC.

\section{EML4-ALK}

The establishment of EML4-ALK fusion gene has again been achieved using molecular techniques.
In 2007, SodA et al. [10], aiming to discover unknown lung cancer driving mutations and genes, managed to generate a retroviral cDNA library from a lung adenocarcinoma specimen. The cDNA library was used to transfect a mouse 3T3 fibroblast cell line. Among the transfected cells, they detected a clone bearing a 3926 base pair CDNA sequence originated from the fusion of the EML4 and ALK genes [10]. The transfected EML4-ALK mutant 3T3 mouse cell line was subcutaneously injected to nude mice and formed primary tumours at the point of the injection. Importantly, the presence of WHI-P154, which is an ALK kinase activity inhibitor, in the cell culture media hampered the proliferation of cells bearing the EML4-ALK fusion gene [11]. Therefore, this mutation may be a suitable target for specific lung cancer treatment. Given that EML4-ALK fusion gene shows high specificity to NSCLC, the specific mutation may be used for the delicate diagnosis of the disease.

Clinical data suggests that $6.7 \%$ of NSCLC patients harbour the EML4-ALK oncogene and this subpopulation is discrete from mutant EGFR or KRAS NSCLC patients. Thus the ALK tyrosine kinase inhibitors may be an effective targeted therapy for a group of patients who previously lacked a specific treatment. Administration of crizotinib has been proven to be beneficial for NSCLC patients, although resistance is developed to the medication in later stages [11].

\section{Summary}

As we move towards the era of personalised medicine, clinical data indicate the fundamental need to screen lung cancer patients in order to determine their specific mutational spectrum and thus adjust their treatment accordingly. Understanding the genetic alterations of NSCLC leads to tailored therapies and effective patient management by minimising the burden of adverse events. The development of innovative agents as well as combinational targeted treatments is thoroughly examined to reduce or nullify drug resistance. Novel lung cancer genes and driving mutations that may be used as drug targets are constantly discovered. Acknowledging the fact that the underlying pathogenesis mechanisms of the disease affect patients' management, efforts should be made to select the suitable agent based on molecular pathway analysis.

\section{Support statement}

N.I. Kanellakis is the recipient of a short-term European Respiratory Society Fellowship. I. Psallidas is the recipient of a RESPIRE2 European Respiratory Society Fellowship (RESPIRE2 - 2015-7160).

\section{Conflict of interest}

None declared. 


\section{References}

1. Jemal A, Bray F, Center MM, et al. Global cancer statistics. $C A$ CancerJ Clin 2011; 61: 69-90.

2. Schiller JH, Harrington D, Belani CP, et al. Comparison of four chemotherapy regimens for advanced non-small-cell lung cancer. N EnglJ Med 2002; 346: 92-98.

3. Kawamoto T, Sato JD, Le A, et al. Growth stimulation of A431 cells by epidermal growth factor: identification of highaffinity receptors for epidermal growth factor by an antireceptor monoclonal antibody. Proc Natl Acad Sci USA 1983; 80: 1337-1341.

4. Rusch V, Baselga J, Cordon-Cardo C, et al. Differential expression of the epidermal growth factor receptor and its ligands in primary non-small cell lung cancers and adjacent benign lung. Cancer Res 1993; 53: Suppl., 2379-2385.

5. Lynch TJ, Bell DW, Sordella R, et al. Activating mutations in the epidermal growth factor receptor underlying responsiveness of non-small-cell lung cancer to gefitinib. N Engl J Med 2004; 350: 2129-2139.
6. Paez JG, Jänne PA, Lee JC, et al. EGFR mutations in lung cancer: correlation with clinical response to gefitinib therapy. Science 2004; 304: 1497-1500.

7. Peters S, Zimmermann S, Adjei AA. Oral epidermal growth factor receptor tyrosine kinase inhibitors for the treatment of non-small cell lung cancer: comparative pharmacokinetics and drug-drug interactions. Cancer Treat Rev 2014; 40: 917-926.

8. Baker NM, Der CJ. Cancer: drug for an 'undruggable' protein. Nature 2013; 497: 577-578.

9. Zimmermann G, Papke B, Ismail S, et al. Small molecule inhibition of the KRAS-PDE $\delta$ interaction impairs oncogenic KRAS signalling. Nature 2013; 497: 638-642.

10. Soda M, Choi YL, Enomoto $M$, et al. Identification of the transforming EML4-ALK fusion gene in non-small-cell lung cancer. Nature 2007; 448: 561-566.

11. Gridelli C, Peters S, Sgambato A, et al. ALK inhibitors in the treatment of advanced NSCLC. Cancer Treat Rev 2014; 40: 300-306 